\title{
AUTHOR INDEX \\ (Volume 6)
}

Abadyan, M. 419

Adeli, H. 113

Adeli, M. 239, 301

Ahmad, A. L. $\quad 81,113$

Ahmad, I. 259

Ahmadpour, A. 349

Ahmed, F. 295

Al Zahrani, S. M. 185

Ali, I. 185

Alubaidy, M. 123

Amin, R. 101, 333

Anwar, M. S. 295

Arshad, M. 357

Arshi, N. 295

Aryanto, D. 153

Bag, B. P. 387

Bahari, A. 301

Balamurugan, J. 251

Bamoharram, F. F. 349

Benayad, A. 409

Botes, M. 395

Brammer, K. S. 541

Cao, S. 265

Cha, M.-H. 225

Chae, S. J. $\quad 59,409$

Chaterjee, P. 379

Chen, C. 145

Chen, M. 287

Chen, P. 325

Chen, Q. 1

Chen, X. 205, 343

Chen, Y. B. 167, 481

Cheng, J. 461

Cho, S. 461

Choi, J. 461

Choi, J.-Y. 59, 409

Choi, K. 225

Choli-Papadopoulou, T. 271
Cloete, T. E. 395

Cohen, S. R. 313

Cui, H. 441

Cui, Y. 145

Dandekar, T. 19

Danikas, M. G. 497

de Kwaadsteniet, M. 395

Deb, A. 379

Dee, C. F. 259

Delavari, H. 41

Dharmaraja, J. 251

Ding, Y.-P. 325

Duong, D. L. 409

Eidelman, O. 313

Eleithy, R. H. 185

Emadi, R. 131

Feldman, Y. 313

Feyen, M. 469

Friedman, H. 313

Ge, H. 287

Ghoreishi, M. 561

Grado-Caffaro, M. 557

Grado-Caffaro, M. A. 557

$\mathrm{Gu}$, B. F. $\quad$ 167, 481

Güneş, F. 409

Han, G. H. 59, 409

Hayashi, J. 583

Hayashi, Y. 583

He, J. 337

He, Y. J. 481

Heravi, M. M. 349

Hitchcock, D. 337

Hong, B. 287

Hosseini, S. M. 301

Hou, Q. R. 167, 481
Huang, B. 287

Huang, S. 205, 343

Hui, W. 145

Husnain, G. 357

Hussain, R. 333

Hwang, S. 101

Hyun, J. 461

Ihm, J. 225

Ishaq, A. 357

Ismail, A. K. 153

Ito, D. 583

Jeong, B. 93

Jia, K.-M. 469

Jiang, H. 589

Jiang, X. 67

Jimbo, T. 583

Jin, C. $\quad 93,455$

Jin, D. 287

Jin, H. 287

Jin, M. 409

Jin, S. 541

Jung, J. 461

Karagiannidis, P. G. 271

Karmakar, B. 173

Kato, S. 583

Katranidis, A. 271

Kazemi, A. 419

Khalid, A. 357

Kim, E. S. $\quad 59,409$

Kim, H. 93, 455

Kim, M. 225

Kishi, N. 583

Kokabi, M. 41

Koo, B. H. 295

Koochi, A. 419

Koshy, J. 279

Kumar, K. S. 139

Kumar, R. 215 
Kumar, S. 295

Kumar, V. R. 279

Lee, C. 93, 455

Lee, C. G. 295

Lee, J. 93

Lee, S. Y. 409

Lee, Y. H. 59, 409

Li, H. 231

Li, L. 287

Li, W.-C. 469

Li, Y. F. 231

Liao, F. 597

Liao, Z.-J. 431

Liew, K. M. 231

Lim, C. W. 363

Liu, F. $\quad 67$

Liu, Y. 47

Logothetidis, S. 271

Lousinian, S. 271

Lu, A.-H. 469

Lu, B. 357

Lu, J. 295

Ma, X. $\quad 75$

Majlis, B. Y. 259

Markowicz, M. 509

Melachroinos, A. 271

Mikiciuk-Olasik, E. 509

Mishra, D. K. 387

Mishra, P. K. 387

Mohapatra, J. 387

Mondal, O. 379

Moshkovith, A. 313

Nambissan, P. M. G. 173

Nguyen, M. C. 225

Nie, H. 205, 343

Nikanjam, F. 575

Noh, K. 541

Othaman, Z. $\quad 153,159$

Pal, M. $\quad 173,379$

Pankratov, E. L. 31
Papadopoulos, G.

271

Park, M. 461

Park, S. 93, 455

Park, S. H. 101, 333, 461

Peng, J. 569

Perfiliev, V. 313

Pitsa, D. 497

Prasad, V. S. $\quad 139,279$

Rahman, A. 185

Rao, A. M. 337

Rapoport, L. 313

Razak, S. I. A. $\quad 81,435$

Riahi-Noori, N. 575

Rosentsveig, R. 313

Roy, B. 173,379

Sadeghi, E. 239

Saito, T. 583

Sakrani, S. 159

Sam, S. P. C. 139

Sarabi, R. S. 239

Sarraf-Mamoory, R. 575

Schüth, F. 469

Sengupta, S. P. 379

Seong, T.-Y. 541

Shakir, I. 333

Shi, F. 145

Shin, H.-J. 59, 409

Shityakov, S. 19

Singh, S. K. 387

Soga, T. 583

Spliethoff, B. 469

Srivastava, O. N. 215

Sultana, I. 333

Sumpono, I. 159

Sun, Y. 1

Szymański, P. 509

Tahmasebipour, G. 561

Tahmasebipour, Y. 561

Tan, B. 123

Tan, S. H. 113

Tavangarian, F. 131
Tenne, R. 313

Tiwari, R. S. 215

Tsai, J. T. H. 431

Venkatakrishnan, K. 123

Viswanathan, B. 251

Wang, C. X. $\quad 441,489$

Wang, G.-H. 469

Wang, H. 1

Wang, J. 265

Wang, T. 67

Wang, X. 287, 589

Wariar, P. R. S. 279

Wibowo, E. 159

Wu, L.-G. 67

Wu, Q.-S. 325

Wu, X. 569

Xu, J. 287

Xue, S. 569

Yan, L. $\quad$ 259, 357

Yan, Z. 569

Yang, G. Z. 441

Yang, K. 337

Yang, S.-W. 597

Yang, Y. 363

Yang, Z. 205, 343

Yao, F. 409

Yao, Z. 205, 343

Yu, H. Q. 231

Yu, Y. 1

Yu, Z. $\quad 75$

Zein, S. H. S. $\quad 81,113,435$

Zhan, L. 569

Zhang, G. 589

Zhang, Q. 265

Zhao, X. 47

Zhong, H. X. 489

Zhou, X. 205, 259, 343

Zhu, Z.-C. 325 